\title{
173. The Mechanism of Isomerization in the Camphor Series by Sulfuric Acid
}

By Takuichi MIKI, Masamoto NISHIKAWA, and Hikoichi HAGIWARA Research Laboratory, Takeda Pharmaceutical Industries, Ltd.

(Comm. by Y. Asahina, M.J.A., Dec. 12, 1955)

Recently, Nishikawa, ${ }^{1>}$ one of the authors, has demonstrated that in the presence of fuming sulfuric acid $\alpha$, trans $\pi$-dihalo- $d$-camphor (I) undergoes isomerization, involving migration of the $\alpha$-halogen atom to the C-6 position as well as inversion of the mother nucleus, to form 6 , trans $\pi$-dihalo-l-camphor (III).

An example of the inversion of the camphor skeleton is the racemization of $d$-camphor in sulfuric acid, ${ }^{2)}$ and the mechanism was first suggested by Asahina. ${ }^{3)}$ Following back his scheme from the product (III) it can easily be understood that the compound before the inversion must be 5 , trans $\pi$-dihalo- $d$-camphor (II).

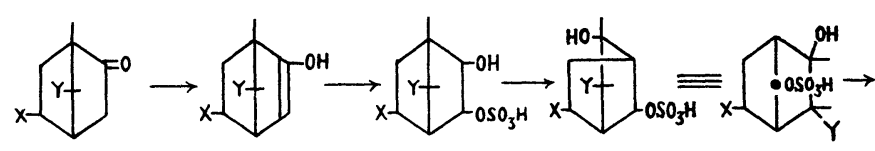

II

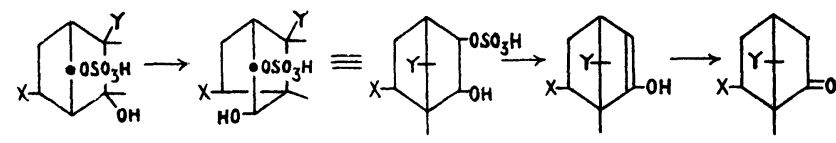

III

$\mathrm{X}$ : Halogen Y: Halomethyl

The authors ${ }^{4)}$ have synthesized 5-bromo-trans $\pi$-chloro- $d$-camphor (II: $\mathrm{X}=\mathrm{Br}, \mathrm{Y}=\mathrm{CH}_{2} \mathrm{Cl}$ ) in the following way:
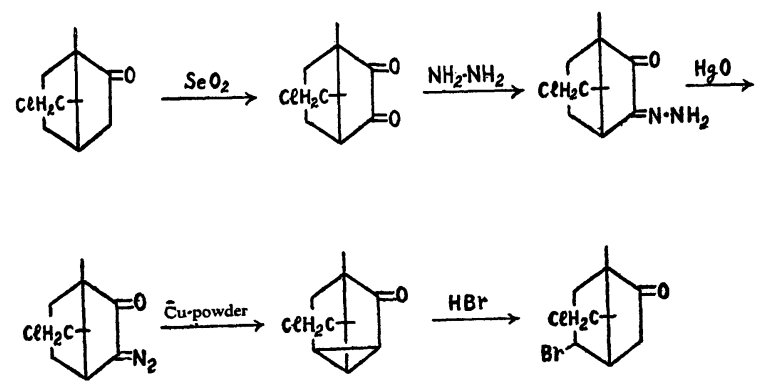

II, however, can not be an intermediate of the reaction since this (II: $\mathrm{X}=\mathrm{Br}, \mathrm{Y}=\mathrm{CH}_{2} \mathrm{Cl}$ ) was found to be quite stable in sulfuric acid, and no isomerization was observed. 
The mechanism of the isomerization given below is proposed by the authors.

The acidic reagent attacks the carbonyl oxygen in I, and the multi-group rearrangement involving the well-known Wagner and Nametkin rearrangements occurs to afford the "non-classical cation" ${ }_{5}$ ) (V) via the intermediate cation (IV). In I, C-6, C-1, C-7, and C-cis $\pi$ lie in one plane, so sterically the migration of the cis $\pi$-methyl group would be more favored than the trans $\pi$-halomethyl group ${ }^{6)}$ to minimize the activation energy. ${ }^{\text {?) }}$

Then in the cation $(\mathrm{V})$, the bond shown by the dotted line weakens with the hydride shifting, and the second multi-group rearrangement sets in. From steric consideration, the methyl group situated in the coplanar direction relative to the weakening bond should preferentially migrate to the adjacent carbon, and the Wagner rearrangement follows via the intermediate cation (VI) to complete the isomerization.

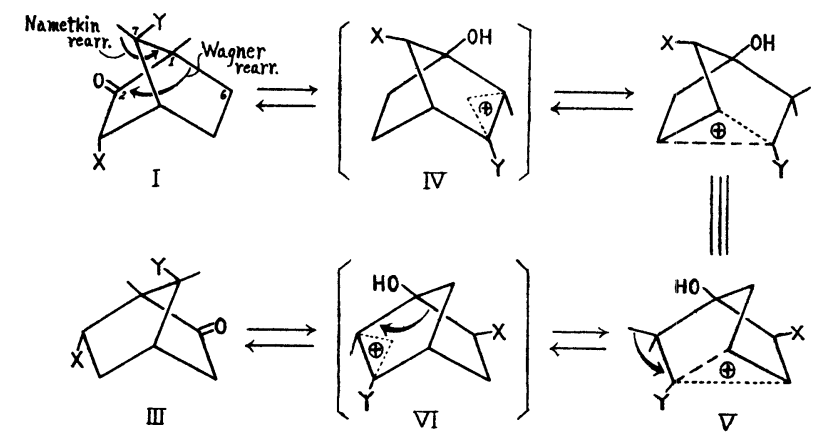

Thus in the final product (III), the halomethyl group should take the trans $\pi$ position, and the mother nucleus undergoes inversion from $d$ - to $l$-type. Since the $\alpha$-halogen in I is consided to take the endo position, ${ }^{8)}$ the $\mathrm{C}-6$ halogen in III should also take the endo position.

Since all the processes of the isomerization described above are reversible, the $\alpha$-halogen compound (I) is of higher energy than $\mathrm{V}$ due to the mutual repulsion ${ }^{9)}$ between $\mathrm{C}=\mathrm{O}$ and the vicinal $\mathrm{C}$-halogen, the equilibrium must be in favor of the latter $(V)$. This was found to be the case.

The above mechanism also explains all the isomerizations in the camphor series in sulfuric acid.

For example, the mechanism of the racemization of trans $\pi$ chloro- $d$-camphor ${ }^{10)}$ (I: $\mathrm{X}=\mathrm{H}, \mathrm{Y}=\mathrm{CH}_{2} \mathrm{Cl}$ ) is as follows: lacking the $\alpha$-halogen atom, I $\left(\mathrm{X}=\mathrm{H}, \mathrm{Y}=\mathrm{CH}_{2} \mathrm{Cl}\right)$ and $\mathrm{V}\left(\mathrm{X}=\mathrm{H}, \mathrm{Y}=\mathrm{CH}_{2} \mathrm{Cl}\right)$ are antipode to each other, and there is no energy difference between them, therefore racemization occurs. 
The cases of $d$-camphor and $\alpha$-chloro- $d$-camphor are also worth mentioning.

$d$-Camphor reacts with sulfuric acid, even under a mild condition, to give rac. camphor-trans $\pi$-sulfonic acid. ${ }^{2)}$ Asahina ${ }^{3)}$ suggested the mechanism of the trans $\pi$-sulfonation which is now generally accepted, and as he pointed out, the racemization might precede to the sulfonation in this reaction.

$\alpha$-Chloro- $d$-camphor (VII), under the same condition, gives $\alpha$ chloro- $d$-camphor-trans $\pi$-sulfonic acid $(\mathrm{X}){ }^{11)}$ This time the racemization of VII is restrained due to the high activation energy to the non-classical cation (VIII), and only the $\pi$-sulfonation occurs. When VII or $\mathrm{X}$ is allowed to stand for a long time in sulfuric acid, the isomerization occurs through the intermediate cations (IX and XI) to give 6-chloro- $l$-camphor-trans $\pi$-sulfonic acid (XII). ${ }^{111)}$

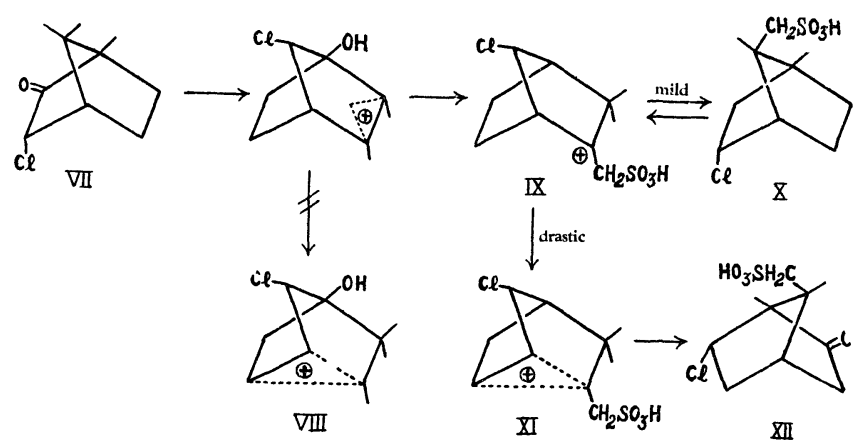

The authors wish to thank Professor Y. Asahina, Dr. S. Kuwada, and Dr. T. Matsukawa for their helpful and stimulating interest in this work.

\section{References}

1) M. Nishikawa: J. Pharm. Soc. Japan, 72, 634, 637, 640, 646 (1952); Proc. Japan Acad., 27, 359 (1951).

2) F. S. Kipping and W. J. Pope: J. Chem. Soc., 63, 549 (1893); 67, 357 (1895).

3) Y. Asahina: Proc. Imp. Acad., 13, 38 (1937).

4) M. Nishikawa and H. Hagiwara: J. Pharm. Soc. Japan (in press).

5) J. D. Roberts et al.: J. Am. Chem. Soc., 72, 3329 (1950); 73, 5009 (1951); 75, 3165,3168 (1953).

6) The considerable proportion of trans $\pi$-halomethyl group migrates to afford $\boldsymbol{\alpha}$, $\omega^{-}$ dihalo- $d$-camphor-trans $\pi$-sulfonic acid. This rearrangement might be favored electrostatically in spite of the steric disadvantages. M. Nishikawa and $H$. Hagiwara: J. Pharm. Soc. Japan, 74, 76, 81 (1954); Proc. Japan Acad., 29, 30 (1953).

7) Synartic acceleration, F. Brown, E. D. Hughes, C. K. Ingold, and J. F. Smith: Nature, 168, 65 (1951).

8) C. W. Shoppee: Chemistry and Industry, 1952, 86. R. C. Cookson: J. Chem. Soc., 1954, 283.

9) The electrostatic repulsion due to dipole-dipole interaction was calculated using E. J. Corey's equation: J. Am. Chem. Soc., 75, 2301 (1953). The value for $\mathrm{I}$ where $\mathrm{X}=\mathrm{Br}$ is $0.91 \mathrm{Kcal} . / \mathrm{mole}$.

10) M. Nishikawa: J. Pharm. Soc. Japan, 72, 634 (1952).

11) J. Ueyanagi: J. Pharm. Soc. Japan, 71, 613 (1951). 